\title{
Anatomical transverse magnetic resonance imaging study of ligaments in palmar surface of metacarpus in Miniature donkey: identification of a new ligament
}

\author{
M.N. Nazem, S.M. Sajjadian \\ Shahid Bahonar University of Kerman, Kerman, Iran
}

[Received: 23 February 2016; Accepted: 14 April 2016]

\begin{abstract}
Background: Palmar region of metacarpus in the horses and donkeys is an important region because of its tendons and ligaments which contribute to stay apparatus. This study was done on forelimbs of 6 healthy Miniature donkeys to detect the tendons, ligaments and their accessories on the palmar surface of metacarpus in this animal.

Materials and methods: Based on that magnetic resonance imaging (MRI) is a good technique to evaluate the soft tissues such as tendons and ligaments, palmar aspects of metacarpus in 6 euthanatised Miniature donkeys were prepared for anatomical and trans-sectional MRI studies to determine the tendons and ligaments in this region.
\end{abstract}

Results: Suspensory ligament, deep digital flexor tendon and its inferior check ligament were similar to them in the horse. Superficial digital flexor tendon (SDFT) in this animal had superior check ligament that was present before the carpal joint. On the other hand in the Miniature donkey there was a second accessory ligament for the SDFT that originated from the proximal of palmar surface of the large metacarpal bone which we named it second accessory ligament of SDFT. This ligament was determined in the MRI images too.

Conclusions: It seems that this ligament helps the Miniature donkey to stay apparatus, supporting more weight and load for a longer period of time and distance which is a specific morphological feature in this animal. (Folia Morphol 2017; 76, 1: 110-116)

Key words: Miniature donkey, superficial digital flexor tendon, second accessory ligament

\section{INTRODUCTION}

Equidae typically support a greater proportion of their body weight with their forelimbs during steady state locomotion $[12,31]$.
The horse has developed tendinous-ligamentous support mechanisms, the stay apparatus, present in both the fore- and hindlimbs, which allow this species to support their body weight with a minimum of mus-

Address for correspondence: M.N. Nazem, Associate Professor of Anatomy, Histology and Embryology, Department of Basic Sciences, School of Veterinary Medicine, Shahid Bahonar University of Kerman, Pazhuhesh Square, Kerman, Iran. P.O. Bax: 76169133. tel: +983433257447, fax: +983433257447, e-mail: nnazem@uk.ac.ir; nasernazem@yahoo.com 
cular effort and reduce muscle fatigue. The efficient use of this apparatus enables the horse to stand for long periods whilst resting. Tendons and ligaments in the palmar surface of metacarpal region are suspensory ligament, superficial digital flexor tendon (SDFT) and deep digital flexor tendon (DDFT) $[4,9,24,28]$.

The suspensory ligament on the palmar aspect of the third metacarpus is one of the most essential anatomical features of the stay apparatus. It serves as the main support for the fetlock, preventing excessive overextension and reducing concussion of the fetlock during locomotion. The superficial and deep digital flexor tendons supplement the suspensory ligament in this function. They are restricted by the accessory ligament between the distal end of the radius and the SDFT (superior check ligament [SCL]) and the accessory ligament between the carpus and the DDFT (inferior check ligament [ICL]). The suspensory ligament and the two digital flexor tendons operate in series. As the fetlock joint is extended by the body weight, the suspensory ligament tightens; this is followed by the extension of the superficial and the deep digital flexor tendons [7, 24].

With magnetic resonance imaging (MRI), an image is obtained based on the magnetic properties of the tissue [29]. MRI enables the detection of small and subtle lesions without the presence of gross structural changes [16].

In equine medicine, several systems are used, ranging from low field (0.2 T) to high field (1.5 T) [29]. Both low-field and high-field MRI give comparable data about abnormal structures, although lesions are more detailed with a high field MRI [21]. MRI is useful for evaluating bone and soft tissues in equine $[2,32,33]$. There is only limited information available on normal MR anatomy of the proximal metacarpal region in the horse $[2,27]$, and the appearance of the proximal metacarpal region has not been described in the Miniature donkey.

The aim of this study was to describe the ligaments and tendons on the palmar aspect of the metacarpus in the Miniature donkey by anatomical dissection and MRI technique.

\section{MATERIALS AND METHODS}

All procedures involving the experimental use of animals were approved by the Animal Ethics Committee, a branch of the Research Council of the Veterinary School in Shahid Bahonar University, Kerman Province, Iran, and administered by the National Animal Ethics Advisory Committee.
Six four to seven-year-old healthy Miniature donkeys euthanatised with a combination of embutramide, mebenzonium iodide, and tetracaine hydrochloride $(4-6 \mathrm{~mL} / 50 \mathrm{~kg})$ injected IV via the vena jugularis for reasons unrelated to the musculoskeletal system and right forelimbs were collected. Then limbs were cut at the junction of the proximal one-third and distal two-thirds of the humerus and frozen within $6 \mathrm{~h}$ after euthanasia. They were stored at $-20^{\circ} \mathrm{C}$ and thawed at room temperature for $24 \mathrm{~h}$ before image acquisition. Low-field imaging was performed sequentially. One freeze-thaw cycle does not influence MRI quality $[22,30]$.

Low-field images were obtained in a $0.27 \mathrm{~T}$ open magnet using a custom-made radiofrequency coil for equine limbs and included motion insensitive 3D T1- and T2-weighted gradient echo (GRE), 2D proton density (PD) spin echo (SE), 2D T2-weighted fast spin echo (FSE) and motion insensitive STIR FSE sequences in transverse plan. The sequences were those used routinely in clinical scanning at the Animal Health Trust. All images were assessed subjectively by the same analyst. Repeatability of image analysis was assessed 6 times in 6 limbs at different levels in 6 donkeys. Three limbs that had been scanned previously were used for producing anatomic specimens in transverse slices to allow for comparison with MRI.

Also three of them that had not been used to produce the transverse slices, were dissected. At first, the skin and superficial fascia, and then deep fascia, were removed. The carpal canal was then opened at the palmar surface by cutting the flexor retinaculum to detect the tendons and structures passing from it. Finally tendons and ligaments at the palmar surface of the metacarpus were dissected from antebrachium to fetlock joint separately.

Normal MR anatomy was described by comparison of images with anatomic specimens and texts $[4,9$, 24]. The same numbering system is used to indicate each anatomic structure in each figure [22].

\section{RESULTS}

\section{Anatomical findings}

The suspensory ligament (or middle interosseus muscle) was a strong tedious band which arose mainly from the proximal end of the cannon bone and the distal row of the carpal bones. It took the form of a uniform ligament which passed between the two splint bones (Fig. 1A, $B ;{ }^{\prime}{ }^{\prime}$ ). This ligament 


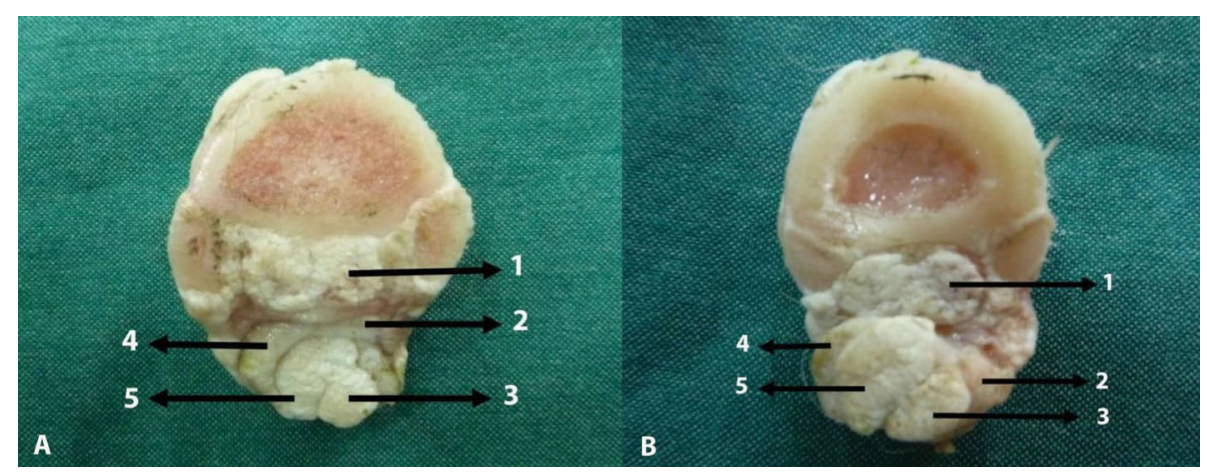

Figure 1. A. Transverse section of metacarpal bones and tendons of this region, obtained $2 \mathrm{~cm}$ and $4.5 \mathrm{~cm}$ (B) distal to the metacarpal joint. Images shown in this figures were obtained in the same limb at two different levels; 1 - suspensory ligament; 2 - second accessory ligament of superficial digital flexor tendon (SDFT); 3 - SDFT; 4 - inferior check ligament; 5 - deep digital flexor tendon.

was divided into two diverging branches which were inserted on the proximal concave abaxial surfaces of sesamoid bones. Each branch was extended a bond obliquely medial and laterally to join the common digital extensor tendon on the dorsal aspect of the first phalanx. The two branches were supported by a small bursa. It is noticeable that no bundles were seen in any limbs between suspensory ligament and DDFT, SDFT, ICL and second accessory ligament of SDFT.

Lateral and medial interosseus muscles were small and each one and lay on each side of the flexor tendons above the fetlock joint.

Superficial digital flexor muscle was triangular in shape; most part of it was covered by the flexor carpi ulnaris. This muscle originated on the medial humeral epicondyle and covered the deep digital flexor muscle. Its belly formed a strong tendon at the level of the carpus, where it was combined with a strong fibrous band, accessory ligament or SCL, which originated from the caudomedial surface of the radius and blended with SDFT.

The SDFT passed distally trough the carpal canal to the palmar aspect of the metacarpus. It was enveloped by the carpal synovial sheath together with the DDFT which was accompanying it from proximal of the carpus to the middle of the metacarpus. SDFT lay mediopalmar to the deep flexor tendon in this region (Fig. $1 \mathrm{~A}, \mathrm{~B} ;{ }^{\prime} 3{ }^{\prime}$ and Fig. $2{ }^{\prime}{ }^{\prime}{ }^{\prime}$ ).

A second accessory ligament of SDFT with strong collagenous band was originated from the deep fascia of the palmar carpal region (deep palmar carpal ligament) at the level of the proximal one eighth of the lateral surface of the metacarpus and joined to the SDFT at this side. It was as thick as the SCL. A common tendon with SDFT was then formed at the upper quarter of the metacarpus and passed to the digits
(Fig. $1 \mathrm{~A}, \mathrm{~B} ;{ }^{\prime} 2$ ' and Fig. $2 ;$ ' 2 '). According to Figures 1 and 2 , there wasn't any connection between SDFT or second accessory ligament of SDFT and DDFT, ICL supensory ligament, and flexor retinaculum. It is noticeable that the origin of this ligament was similar to origin of ICL.

The deep digital flexor muscle consisted of three heads. The humeral head originated from the medial epicondyle of the humerus and run to the caudal surface of the radius and was almost completely covered by the superficial flexor muscle. The ulnar head had an origin on the medial aspect of the olecranon. Initially, it lay superficially between the flexor and extensor carpi ulnaris muscles and then became tendinous to pass distally between the humeral head and the extensor muscle of the ulnar carpal bone. It finally joined with the main tendons near the carpus. The radial head arose as a flat muscle belly from the caudomedial aspect of the radius at about the middle third of the forearm. Its tendon blended with the chief tendon near the distal end of the radius. The tendons were then constituted the DDFT (Fig. 1A, B; ' 5 ' and Fig. $2 ;$ ' 3 '). It passed over the flexor aspect of the carpus, medial to the accessory carpal bone. Distal to the carpal joint, this tendon became roundish and was in close relation with the SDFT but there wasn't connection between them. In the upper quadrant of the metacarpus, an accessory ligament or ILC, that was a continuation of the deep palmar carpal ligament after the carpal joint, and was making from collagenous strong bands, connected to it. It was located between the interosseus and the DDFT (Fig. 1A, $B ;$ ' 4 ' and Fig. 2 ; ' 4 '). During the carefully dissection, no bundles were seen in any limbs between ICL and flexor retinaculum, or between ICL and either SDFT or DDFT. In other words, ICL only merged with DDFT. 


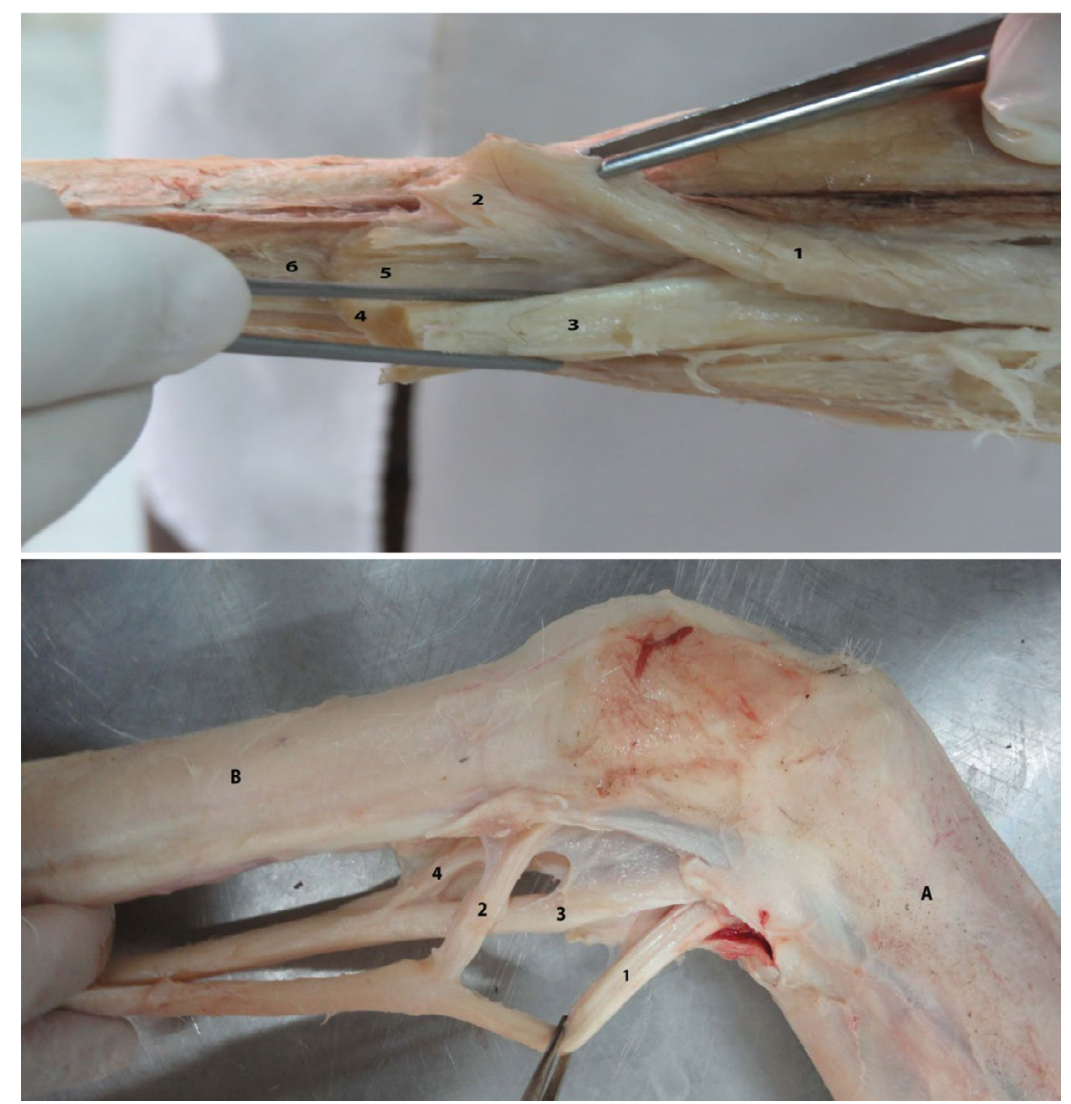

Figure 2. A. Radius; B. Metacarpus; Ligaments that origin from deep fascia of palmar surface which join to the superficial digital flexor tendon and deep digital flexor tendon in palmar view of metacarpus. 1 - superficial digital flexor tendon; 2 - second accessory ligament of superficial digital flexor tendon; 3 - deep digital flexor tendon; 4 - inferior check ligament; 5 - deep palmar carpal ligament; 6 - suspensory ligament.

In the distal quarter of the metacarpus, above the gliding surface of the sesamoid bones, the superficial tendon enclosed the deep tendon - like a cuff (manica flexoria). The two tendons were thus intimately associated as they continued over the palmar aspect of the fetlock.

\section{MRI findings}

The medulla of the third metacarpal bone had heterogeneous intermediate signal intensity on T1 and T2 GRE images and the signal intensity increased distally. The cortex had homogeneous low signal intensity. The thickness of the dorsal, palmar, medial, and lateral cortices increased from proximal to distal. The smoothness of the periosteum was often difficult to evaluate at the origin of the suspensory ligament, because both the cortex and the suspensory ligament had low signal intensity. The cortices of the second and fourth metacarpal bones had low signal intensity and were thicker on the abaxial aspect of the bones.

The majority of the fibres of the suspensory ligament originated from the proximal palmar cortex of the third metacarpal bone, but there were additional fibres originating from the palmar aspect of the third carpal bone. The suspensory ligament was a bilobed structure proximally. The two lobes merged a few distance distal to the carpometacarpal joint. The suspensory ligament was surrounded by loose connective tissue, which had intermediate to high signal intensity in all image sequences. Low signal intensity bundles were seen between the proximal aspect of the suspensory ligament and the palmar aspect of the third metacarpal bone at a variable proximodistal location in all donkeys. Similar bundles were seen between the suspensory ligament and the accessory ligament of the deep digital flexor tendon in all donkeys. From $1 \mathrm{~cm}$ distal to the site where the fibres from the palmar carpal ligament and the fibres from the third carpal bone join, the accessory ligament of the deep digital flexor tendon, second accessory ligament of superior digital flexor tendon and the suspensory ligament were clearly separated (Fig. 3A, B; ' 1 ').

The deep digital flexor tendon had a triangular or tear-drop shape, with its apex pointing medially at the level of the carpometacarpal joint; it became 


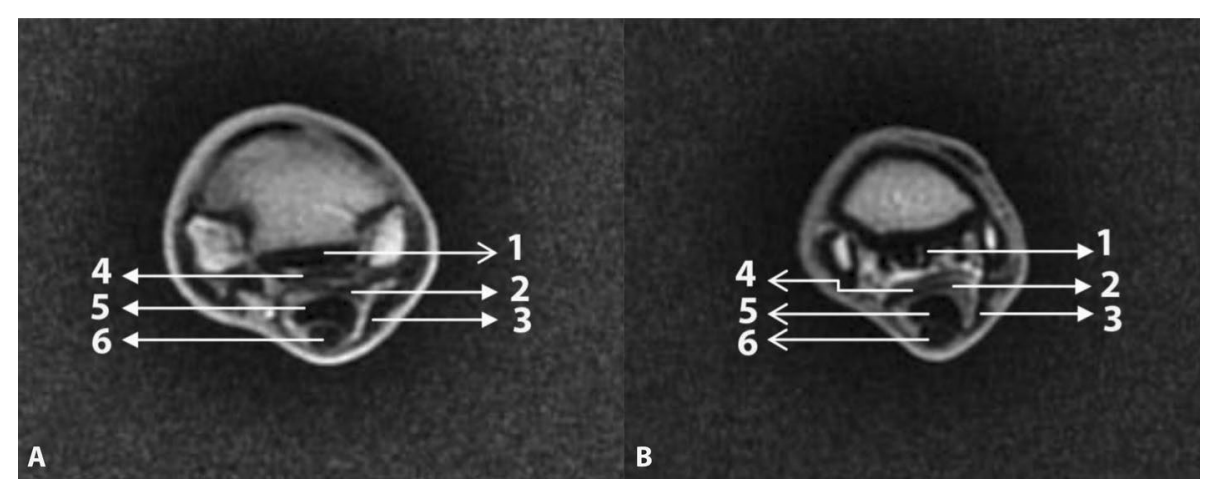

Figure 3. Transverse low-field magnetic resonance images obtained $2 \mathrm{~cm}(\mathbf{A})$ and $3 \mathrm{~cm}(\mathbf{B})$ distal to the carpometacarpal joint. A, B. Transverse proton density image. Medial is to the left and dorsal to the top; 1 - suspensory ligament; 2 - second accessory ligament of superficial digital flexor tendon (SDFT); 3 - flexor retinaculum; 4 - inferior check ligament; 5 - deep digital flexor tendon; 6 - SDFT.

more round distally (Fig. 3A, B; '5'). The accessory ligament of the deep digital flexor tendon was a distal continuation of the palmar carpal ligament and had fibres originating from the palmar aspect of the third carpal bone that was similar to second accessory ligament of SDFT. At the level of the carpometacarpal joint it had heterogeneous intermediate signal intensity, ill-defined margins and was located dorsal to the deep digital flexor tendon. Distally, the margins became better defined and the signal intensity more homogeneous. On T1and T2-weighted GRE sequences it had low signal intensity in PD SE, FSE, and STIR sequences. As a result, on transverse images the accessory ligament of the deep digital flexor tendon had homogeneous or heterogeneous signal intensity, corresponding to the location of the bands on the sagittal images (Fig. 3A, $B$; ' 4 ').

The superficial digital flexor tendon had uniform low signal intensity and smooth, well-defined margins. On transverse images, it had tear-drop shaped, with its lateral aspect more narrow and pointed. Further distally, it became an oval shape (Fig. 3A, B; ' 6 '). A ligament that we named the second accessory ligament of the SDFT was a distal continuation of the palmar carpal ligament and had fibres that originated from the palmar aspects of the third carpal bone. This new ligament had heterogeneous intermediate signal intensity, ill-defined margins and was situated dorsal to the deep digital flexor tendon near the accessory ligament of DDFT (Fig. 3A, B; '2').

\section{DISCUSSION}

In quadrupeds, the centre of gravity is located in the body centre of a standing animal and shifts around the centre during locomotion [1]. The efficient use of the stay apparatus enables the horse to stand still for a long period whilst resting [6]. The major role of the forelimb is to support the body weight [24]. A greater proportion (60\%) of the body weight of the horse is borne on the front legs [18]. However, the load at the stance phase in Thoroughbred racehorses was recorded as $120 \%$ of the body weight during trot [11], $120-150 \%$ of the body weight during canter [19] and $170 \%$ of the body weight during gallop [15]. During walking, trotting and galloping, the forelimbs bear more weight than the hindlimbs [10].

The stay apparatus prevents excessive dorsal flexion of the fetlock. It also limits palmar flexion by means to the common digital extensor tendon and diminishes concussion [24, 28].

The large (third) metacarpal bone (MC2) bears most of the weight, but the small second and fourth bones (splint bones) also bear weight from the carpus even though they have no distal means of support [6]. Additionally, the MC3 in the stance phase is primarily loaded in axial compression [20].

The carpus is predisposed to effortless weight bearing since the long axis of the radius and the cannon bone are approximately in the same vertical line. It is prevented from bucking forwards by the lacertus fibrosus which inserts on the caudal aspect of the carpus, the ligaments of the accessory bone and the accessory ligaments of the superficial and deep digital flexor tendons (SCL and ICL) [6].

Suspensory ligament, SDF and DDF muscles are all active during stance and are thought to support body weight [12].

Similar studies have concluded that the interosseus-sesamoidean suspensory apparatus absorbs the 
initial concussion when the hoof strikes the ground. As the fetlock joint starts to hyperextend, the digital flexor tendons act to fetlock joint angulation [6]. The primary function of the suspensory ligament is to provide the proximal support of the fetlock $[4,24]$. The tension of the suspensory ligament on the sesamoid bones that is transmitted to the palmar surface of the proximal and middle phalanges by the sesamoid ligaments has an important role to body weight tolerance [6].

The suspensory ligament and two digital flexor tendons operate in series. As the fetlock joint is extended by the body weight, the suspensory ligament tightens, this is followed by the superficial and then deep digital flexor tendons $[26,28]$. Digital flexors by their respective tendons provide the thoracic limb with a series of ligaments that act as a passive of the stay apparatus that provides primary resistance to over extension of joints during the support phase of the gait $[6,8]$.

In this study, suspensory ligament and its extensor branches were similar to the horse. According to our findings, ICL was merged with DDFT in the upper quadrant of the metacarpus while it is in the middle third of the metacarpus in the horse $[4,6]$. Other properties of the deep digital flexor muscle and its tendon in the Miniature donkey were similar to those in the horse $[4,6,24,25]$. Contrary to our results and some previous studies $[4,6,24,25]$, Nagy and Dyson [23] reported fibrous bundles between the ICL and lateral aspect of SDFT and DDFT in the horse. According to their findings, the bundles were clearly separated from the flexor retinaculum. They notified no bundles were seen between ICL and the flexor retinaculum biaxially, or between the medial aspect of ICL and either the SDFT or the DDFT [23]. As mentioned in the results, we didn't find any of these connections in our anatomical our MRI findings. According to Figure $1 \mathrm{~A}\left({ }^{\prime} \mathrm{L}^{\prime}, \mathrm{\prime}^{\prime} \mathrm{\prime}^{\prime}\right)$, at the proximal extremity of metacarpus, the ICL was next to the second accessory ligament but there was no connection between them. During their continuation to the distal, they took distance from each other (Fig. 1B; ' 2 ', ' 4 ').

Although properties of SDFT in this study were similar to those observed in the horse $[4,6,24]$, there is no report about the second accessory ligament of SDFT till now. It seems that, this ligament developed in the Miniature donkey as a reinforcing ligament.

The main advantage of MRI over radiography and diagnostic ultrasound is that it provides both anatomical and physiological information in multiple planes. Most of the soft tissues surrounding the carpometacarpal and metacarpophalangeal joint can readily be identified even with a low field system [17]. We used low field system in this study and found this new ligament. Our MRI findings about suspensory ligament, SDFT, DDFT and accessory ligament of DDFT were similar to previous researchers' findings in the horse $[3,5,13,14,22]$.

\section{CONCLUSIONS}

It seems that there is a specific morphological feature in Miniature donkey that is associated with running long distance and with supporting more weight. On the other hand, this accessory ligament of the SDFT which is found in this breed specifically, can provide additional stabilisation and support of SDFT.

\section{Acknowledgements}

The authors would like to thank Dr. Tahmouresi for preparation the figures. This research was financially supported by a grant (No: 1/06/1392) from the Technology and Research Council of Shahid Bahonar University of Kerman, Iran.

\section{REFERENCES}

1. Alrtib AM, Philip CJ, Abdunnabi $\mathrm{AH}$, et al. Morphometrical study of bony elements of the forelimb fetlock joints in horses. Anat Histol Embryol. 2013; 42(1): 9-20, doi: 10.1111/j.14390264.2012.01158.x, indexed in Pubmed: 22571501.

2. Bischofberger AS, Konar M, Ohlerth $S$, et al. Magnetic resonance imaging, ultrasonography and histology of the suspensory ligament origin: a comparative study of normal anatomy of warmblood horses. Equine Vet J. 2006; 38(6): 508-516, doi: 10.2746/042516406×156109, indexed in Pubmed: 17124840.

3. Brokken MT, Schneider RK, Sampson SN, et al. Magnetic resonance imaging features of proximal metacarpal and metatarsal injuries in the horse. Vet Radiol Ultrasound. 2007; 48(6): 507-517, doi: 10.1111/j.17408261.2007.00288.x, indexed in Pubmed: 18018721.

4. Budras KID, Sack WO, Rock S. Anatomy of the horse. 5th ed. Frankfurt, Germany: Schlutersche. 2009: 8-11.

5. Cillán-García E, Milner PI, Talbot A, et al. Deep digital flexor tendon injury within the hoof capsule; does lesion type or location predict prognosis? Vet Rec. 2013; 173(3): 70, doi: 10.1136/vr.101512, indexed in Pubmed: 23736518.

6. De Lahunta A, Habel RE. Applied veterinary anatomy. 3rd ed. Philadelphia, USA: WB Saunders. 1986: 104-105.

7. Dyson S, Murray R, Schramme M, et al. Magnetic resonance imaging of the equine foot: 15 horses. Equine Vet J. 2003; 35(1): 18-26, doi:10.2746/042516403775467531, indexed in Pubmed: 12553458. 
8. Dyson SJ, Kidd L. A comparison of responses to analgesia of the navicular bursa and intra-articular analgesia of the distal interphalangeal joint in 59 horses. Equine Vet J. 1993; 25(2): 93-98, doi: 10.1111/j.2042-3306.1993. tb02915.x, indexed in Pubmed: 8467786.

9. Getty R. Sisson and Grossman's the anatomy of the domestic animals, 5th ed. Philadelphia: W.B. Saunders, 1990

10. Gustås $P$, Johnston $C$, Roepstorff $L$, et al. Relationships between fore- and hindlimb ground reaction force and hoof deceleration patterns in trotting horses. Equine Vet J. 2004; 36(8): 737-742, doi: 10.2746/0425164044848136, indexed in Pubmed: 15656507.

11. Hjertén G, Drevemo S. Semi-quantitative analysis of hoofstrike in the horse. J Biomech. 1994; 27(8): 997-1004, doi: 10.1016/0021-9290(94)90216-x, indexed in Pubmed: 8089167.

12. Hudson PE, Corr SA, Payne-Davis RC, et al. Functional anatomy of the cheetah (Acinonyx jubatus) forelimb. J Anat. 2011; 218(4): 375-385, doi:10.1111/j.14697580.2011.01344.x, indexed in Pubmed: 21332715.

13. Kasashima $Y$, Kuwano $A$, Katayama $Y$, et al. Magnetic resonance imaging application to live horse for diagnosis of tendinitis. J Vet Med Sci. 2002; 64(7): 577-582, doi: 10.1292/jvms.64.577, indexed in Pubmed: 12185310.

14. Kasashima $Y$, Kuwano A, Katayama $Y$, et al. Magnetic resonance imaging application to live horse for diagnosis of tendinitis. J Vet Med Sci. 2002; 64(7): 577-582, doi: 10.1292/jvms.64.577, indexed in Pubmed: 12185310.

15. Kingsbury HB, Quddus MA, Rooney JR, et al. A laboratory system for production of flexion rates and forces in the forelimb of the horse. Am J Vet Res. 1978; 39(3): 365-369, indexed in Pubmed: 637385.

16. Kraft SL, Gavin P. Physical principles and technical considerations for equine computed tomography and magnetic resonance imaging. Vet Clin North Am Equine Pract. 2001; 17(1): 115-30, vii, indexed in Pubmed: 11488039.

17. Martinelli MJ, Kuriashkin IV, Carragher BO, et al. Magnetic resonance imaging of the equine metacarpophalangeal joint: three-dimensional reconstruction and anatomic analysis. Vet Radiol Ultrasound. 1997; 38(3): 193-199, doi: 10.1111/j.1740-8261.1997.tb00840.x, indexed in Pubmed:9238790.

18. McDuffee LA, Stover SM, Coleman K. Limb loading activity of adult horses confined to box stalls in an equine hospital barn. Am J Vet Res. 2000; 61(3): 234-237, doi: 10.2460/ ajvr.2000.61.234, indexed in Pubmed: 10714511.

19. Merkens HW, Schamhardt HC, Van Osch GJ, et al. Ground reaction force patterns of Dutch warmblood horses at normal trot. Equine Vet J. 1993; 25(2): 134-137, doi: 10.1111/j.2042-3306.1993.tb02923.x, indexed in Pubmed: 8467772.

20. Merritt JS, Pandy MG, Brown NAT, et al. Mechanical loading of the distal end of the third metacarpal bone in horses during walking and trotting. Am J Vet Res. 2010; 71(5): 508-514, doi: 10.2460/ajvr.71.5.508, indexed in Pubmed: 20433375.

21. Murray RC, Mair TS, Sherlock CE, et al. Comparison of high-field and low-field magnetic resonance im- ages of cadaver limbs of horses. Vet Rec. 2009; 165(10): 281-288, doi: 10.1136/vr.165.10.281, indexed in Pubmed: 19734560.

22. Nagy A, Dyson S. Magnetic resonance anatomy of the proximal metacarpal region of the horse described from images acquired from low- and high-field magnets. Vet Radiol Ultrasound. 2009; 50(6): 595-605, doi: 10.1111/j.1740-8261.2009.01589.x, indexed in Pubmed: 19999342.

23. Nagy A, Dyson S. Anatomical, magnetic resonance imaging and histological findings in the accessory ligament of the deep digital flexor tendon of forelimbs in nonlame horses. Equine Vet. J. 2011; 43(3): 309-316, doi: 10.1111/j.2042-3306.2010.00217.x, indexed in Pubmed: 21492208.

24. Nickel R, Schummer A, Seiferle E. The anatomy of the domestic animals. 2nd ed. Verlag Paul Parey, Berlin, Humburg, Germany. 1973; 3: 77-99.

25. Murray R, Roberts B, Schramme M, et al. Quantitative Evaluation of Equine Deep Digital Flexor Tendon Morphology using Magnetic Resonance Imaging. Vet Radiol Ultrasound. 2004; 45(2): 103-111, doi: 10.1111/j.17408261.2004.04017.x.

26. Reis A, Baccarin R. The cross-sectional area of the superficial digital flexor tendon of trained and untrained Thoroughbred racehorses. Ciência Rural. 2010 ; 40(8): 1786-1790, doi: 10.1590/s010384782010000800018.

27. Sampson SN, Tucker RL. Magnetic resonance imaging of the proximal metacarpal and metatarsal regions. Clin Tech Equine Pract. 2007; 6: 78-85.

28. Tony WL, Luis C, Soslowsky $\sqcup$. Biomechanics of tendon injury and repair. J Biomech. 2004; 37: 865-877.

29. Tucker RL, Sande RD. Computed tomography and magnetic resonance imaging of the equine musculoskeletal conditions. Vet Clin North Am Equine Pract. 2001; 17(1): 145-57, vii, indexed in Pubmed: 11488041.

30. Widmer WR, Buckwalter KA, Hill MA, et al. A technique for magnetic resonance imaging of equine cadaver specimens. Vet Radiol Ultrasound. 1999; 40(1): 10-14, doi: 10.1111/j.1740-8261.1999.tb01832.x, indexed in Pubmed: 10023989.

31. Witte TH, Knill K, Wilson AM. Determination of peak vertical ground reaction force from duty factor in the horse (Equus caballus). J Exp Biol. 2004; 207(Pt 21): 3639-3648, doi: 10.1242/jeb.01182, indexed in Pubmed: 15371472.

32. Zubrod CJ, Schneider RK, Tucker RL, et al. Use of magnetic resonance imaging for identifying subchondral bone damage in horses: 11 cases (1999-2003). J Am Vet Med Assoc. 2004; 224(3): 411-418, doi: 10.2460/ javma.2004.224.411, indexed in Pubmed: 14765802.

33. Zubrod C, Schneider R, Tucker R. Use of magnetic resonance imaging to identify suspensory desmitis and adhesions between exostoses of the second metacarpal bone and the suspensory ligament in four horses. J Am Vet Med Assoc. 2004; 224(11): 1815-1820, doi:10.2460/ javma.2004.224.1815. 